\title{
A Cost Effective Method for Determining the Position of Mine Haul Road Defects From the Road Edge
}

\author{
doi:10.3991/ijim.v4i1.1067 \\ F. Sokolic ${ }^{1,2}$, M. Rawlins ${ }^{2}$ and K.J. Duffy ${ }^{1}$ \\ ${ }^{1}$ Durban University of Technology, Durban, South Africa \\ 2 Energy Combustion Services, Durban
}

\begin{abstract}
This paper describes a simple method for estimating haul road defect positions from alongside roads. It is a method that is cheap and easy to implement, needing only a minimal amount of field equipment and training. Observers are required to estimate the bearing and distance to a defect, and to record their location using a GPS receiver. All further processing is automated and can be done entirely within a spatially-enabled database management system such as Microsoft SQL Server or PostgreSQL.
\end{abstract}

Index Terms-rangefinder, GPS, position measurement, road maintenance.

\section{INTRODUCTION}

In open-pit mines the maintenance of haul roads is an ongoing and expensive process with major implications for haul truck fuel efficiency. Not only do poorly maintained roads lead to increases in fuel consumption, they also negatively impact on the operational efficiency of the mine itself. To counter this, regular monitoring and maintenance of mine haul roads is carried out. In a typical haul road monitoring and maintenance programme, observers monitor road conditions on an ongoing basis, recording the type, severity and position of all road defects. This information is then passed on to maintenance crews who are responsible for repairing the defects. All information on defect detection, monitoring and repair is typically recorded in a computerised management system.

A major problem experienced by haul road maintenance personnel in operational open-pit mines is that the activities of large haul trucks often makes it too dangerous for them to stand on the road itself when recording the locations of road defects such as potholes. To reduce the risks to the observer road defects must be located from the edge of roads. A method has been devised whereby the defects are recorded from the side of the road, with the bearing and distance to the defect being estimated by the observer.

The proposed method does not rely on expensive equipment such as laser range-finders or laser theodolites which can cost up to $\$ 12,000$ [1]. Rather, the method described here is a low-technological solution requiring only a hand-held Global Positioning System (GPS) receiver and, optionally, a hand held compass.

\section{Methodology}

The initial step in determining the position of a road defect sees an observer standing alongside the road and estimating the distance and bearing to the defect. The position at which the observer is standing, along with the bearing and distance to the defect define a defect vector from which the true position of the defect can be determined. This is done using a series of steps in which the defect bearing is first corrected using the orientation of road, and then, together with the vector length, is used to calculate $\mathrm{x}$ and $\mathrm{y}$ offset values that are applied to the defect vector origin to give the actual position of the defect itself.

The complete procedure for calculating the position of the defect is based on trigonometrical principles and is made up of three basic steps:

1. Measure the defect vector

2. Convert the defect vector bearing from a relative to a true bearing

3. Calculate the coordinates of the defect

The first step is performed on-site in the mine, while steps 2 and 3 can either be performed on-site or as part of a post-processing procedure.

\section{A. Step 1: Measure the defect vector}

Road defects are recorded by observers equipped with a GPS receiver and possibly also a compass. When a defect is located, the observer stands alongside the road and estimates the distance and bearing to the defect as shown in Figure 1.

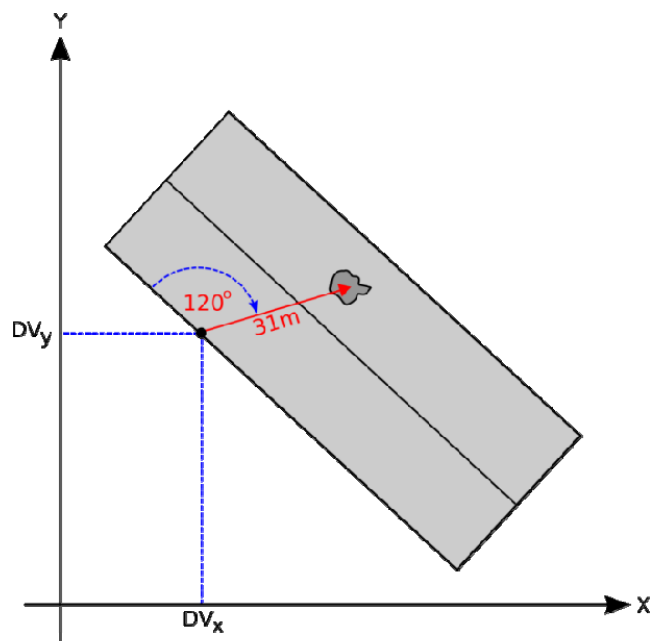

Figure 1. Measurement of a defect position from alongside the road. The bearing $\left(120^{\circ}\right)$, distance (31 metres) and origin (observer location $\left.\left[\mathrm{DV}_{\mathrm{X}} \mathrm{DV} \mathrm{V}_{\mathrm{Y}}\right]\right)$ define a defect vector, shown in red. 
The estimated distance to the defect is given in metres while the bearing is measured in degrees measured in a clockwise direction relative to the road alongside which the observer is standing. Using a GPS the observer also records the current position, thereby defining the defect vector origin. These three measurements, namely the defect distance, defect bearing, and GPS capture position make up a defect vector that provides all the information required to determine the true position of the defect.

\section{B. Step 2: Convert the defect bearing from relative to true}

In this step the true bearing (i.e. relative to True North) of the road is determined. The road bearing is then applied as a correction to the defect vector bearing to convert it to a true bearing.

The first task in this process is to determine the geometric relationship between the defect vector and the road next to which it was measured. This is essential because two different defect bearings are possible depending on whether the observer is standing on the left- or the righthand side of the road. These two bearings will differ by $180^{\circ}$. A point to note here is that the defect bearing is measured using the geographic convention of angle measurement, in which angles are measured in a clockwise direction (Figure 2). The arithmetic system of angle measurement, on the other hand, sees angles increasing in an anticlockwise direction. This difference between the two systems is important as the trigonometrical steps that follow use arithmetic and not geographic angles. However, the advantage of recording defect bearings as geographic angles is that this allows them to be measured in the field using a simple hand-held compass.

The process of determining the road bearing starts in a Geographic Information System (GIS) where the road centre-lines are represented by a series of edges (or lines) and nodes (or points) which together make up a network model of the mine haul road system (Figure 3).
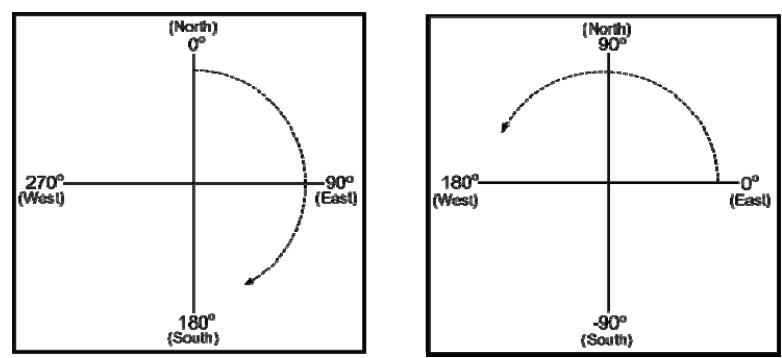

Figure 2. Measurement of geographic angles (left) and arithmetic angles (right).

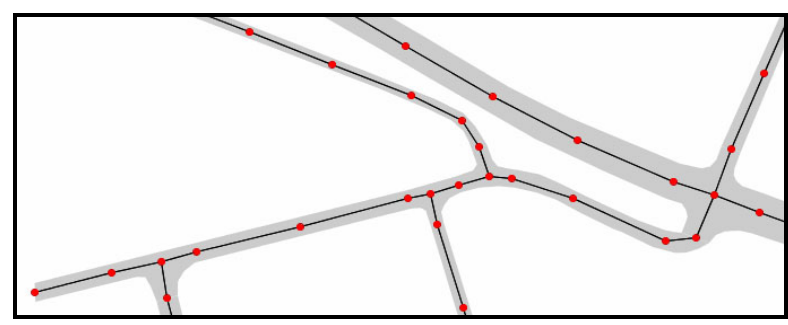

Figure 3. Network model of haul roads (shown in grey) as implemented in a GIS. Nodes (red circles) are connected by edges (black lines). Each edge has one start node and one end node that determine the direction in which the edge is traversed.
Once the defect vector origin is imported into the GIS a simple spatial query is used to select the closest network edge (Figure 4). The nearest edge is defined by start and end nodes, the coordinates of which can be determined by the GIS. Using trigonometrical principles it is then a fairly simple process to calculate the bearing of the road using these start and end node coordinates. Before doing so, however, it should be ensured that the start and end nodes define a right-to-left traversal of the road relative to the defect vector origin. In other words, when viewing the nodes from the defect vector origin the start node should always be to the right of the end node. This ensures that the calculated road bearings will always increase in an anticlockwise direction, as would be expected with arithmetic angles. If it is found that the nodes define a left-toright traversal of the road then the nodes must be swapped around, i.e. the start node becomes the end node and vice versa. With the geometry of the start and end nodes known, the bearing of the road can be calculated and applied to the defect vector bearing (Figure 5).

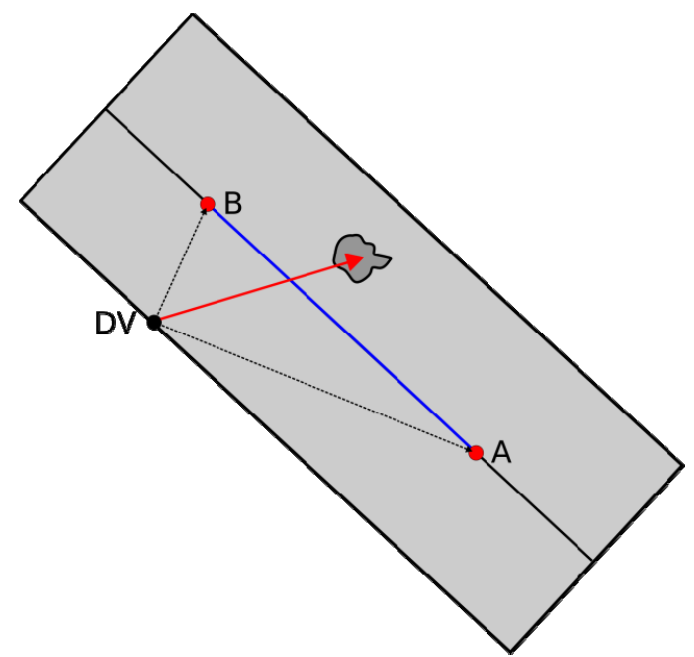

Figure 4. A GIS is used to identify the closest network edge (shown in blue) to the defect vector origin. The start (A) and end (B) nodes of the closest edge are indicated with red circles.

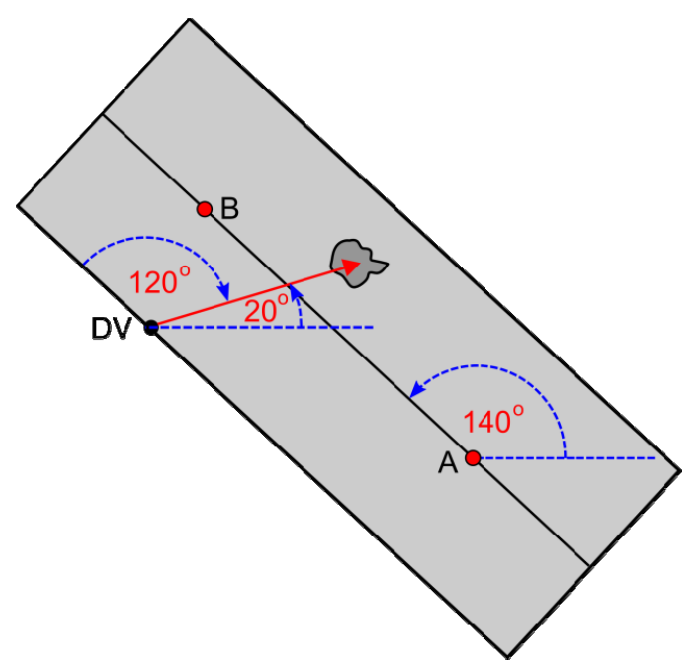

Figure 5. The coordinates of the start and end nodes allow the true bearing of the road to be calculated $\left(140^{\circ}\right)$. The difference between the road bearing and the relative defect vector bearing gives the true bearing of the defect $\left(20^{\circ}=140^{\circ}-120^{\circ}\right)$. True bearings are given using arithmetic angles, where $0^{\circ}$ is equivalent to $90^{\circ}$ geographic, i.e. East. 
The procedure outlined above can be implemented as follows:

- Import the coordinates of the defect vector (DV) origin into a GIS. If necessary the coordinates should be converted from geographic longitude/latitude values to metres-based Cartesian coordinates. These are denoted as the coordinate pair $\left(\mathrm{DV}_{\mathrm{X}} ; \mathrm{DV}_{\mathrm{Y}}\right)$

- Find the network edge (E) closest to the defect vector origin

- Obtain the coordinates of the start (A) and end (B) nodes of this nearest edge. These are denoted as coordinate pairs $\left(\mathrm{A}_{\mathrm{X}} ; \mathrm{A}_{\mathrm{Y}}\right)$ and $\left(\mathrm{B}_{\mathrm{X}} ; \mathrm{B}_{\mathrm{Y}}\right)$ and should also be metres-based Cartesian coordinates

- Calculate the bearings from the defect vector origin to the start and end nodes, using ATan2 to ensure that values in the range $\pm 180^{\circ}$ are obtained:

$\mathrm{A}_{\text {bearing }}=\mathrm{ATan} 2\left(\mathrm{~A}_{\mathrm{X}}-\mathrm{DV}_{\mathrm{X}}, \mathrm{A}_{\mathrm{Y}}-\mathrm{DV} \mathrm{V}_{\mathrm{Y}}\right)$

$\mathrm{B}_{\text {bearing }}=\mathrm{ATan} 2\left(\mathrm{~B}_{\mathrm{X}}-\mathrm{DV} \mathrm{V}_{\mathrm{X}}, \mathrm{B}_{\mathrm{Y}}-\mathrm{DV}_{\mathrm{Y}}\right)$

- Make sure that the start node is to the right of the end node:

If $\left(\mathrm{A}_{\text {bearing }}-\mathrm{B}_{\text {bearing }}<-180^{\circ}\right)$ OR $\left(\mathrm{A}_{\text {bearing }}-\mathrm{B}_{\text {bearing }} \geq 0^{\circ}\right.$ AND $A_{\text {bearing }}-B_{\text {bearing }}<180^{\circ}$ ) then swap $A$ and $B$ (i.e. set $A_{X}=B_{X}, A_{Y}=B_{Y}, B_{X}=A_{X}, B_{Y}=A_{Y}$ )

- Calculate the bearing of the network edge: $\mathrm{E}_{\text {bearing }}=\mathrm{ATan} 2\left(\mathrm{~B}_{\mathrm{X}}-\mathrm{A}_{\mathrm{X}}, \mathrm{B}_{\mathrm{Y}}-\mathrm{A}_{\mathrm{Y}}\right)$

- Subtract the edge bearing from the defect vector bearing to give the true bearing of the defect $\left(\right.$ Def $_{\text {bear- }}$ ing):

Def $f_{\text {bearing }}=\mathrm{DV}_{\text {bearing }}-\mathrm{E}_{\text {bearing }}$

\section{Step 3: Calculate the true coordinates of the defect}

With the true bearing to the defect now known, it is a simple matter to calculate the coordinates of the defect using trigonometry. In this step the offsets of the defect along the $\mathrm{x}$ - and $\mathrm{y}$-axes are calculated and added to the coordinates of the defect vector origin (Figure 6). The resulting coordinate pair $\left(\operatorname{Def}_{\mathrm{X}}\right.$; $\left.\operatorname{Def}_{\mathrm{Y}}\right)$ represents the true position of the defect in the Cartesian coordinate system being used. If necessary these defect coordinates can be converted into longitude/latitude coordinates.

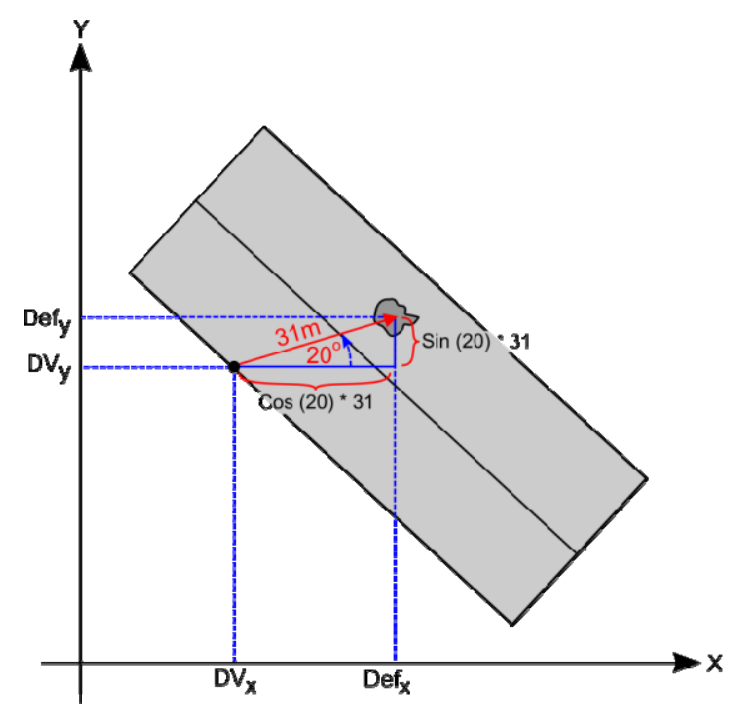

Figure 6. The offsets of the defect on the $\mathrm{x}$ - and $\mathrm{y}$-axes are calculated using the cosine and sine rules. The offsets are added to the coordinates of the defect vector origin $\left(\mathrm{DV}_{\mathrm{X}} ; \mathrm{DV}_{\mathrm{Y}}\right)$ to give the true coordinates of the defect $\left(\right.$ Def $_{X} ;$ Def $\left._{Y}\right)$
These steps can be implemented as follows:

- Calculate the true coordinates of the defect:

$\operatorname{Def}_{\mathrm{X}}=\mathrm{DV} \mathrm{V}_{\mathrm{X}}+\left(\operatorname{Cos}\left(\mathrm{Def}_{\text {bearing }}\right) * \mathrm{DV}_{\text {length }}\right)$

$D e f_{Y}=D V_{Y}+\left(\operatorname{Sin}\left(\right.\right.$ Def $\left.\left._{\text {bearing }}\right) * D V_{\text {length }}\right)$

- [Optional] Reproject the defect coordinates to longitude/latitude values

\section{DISCUSSION}

Recording haul road defects in an operational mine holds risks for the observers due to the activities of mining equipment and haul trucks. The method presented in this paper attempts to mitigate these risks by providing a means of recording defect positions while standing in a safe position next to the road.

When designing and implementing such a system it is important to understand the accuracy levels that can be achieved. The proposed system can be split into two phases, namely a human phase and an automated phase. The human phase, which is where potential errors and inaccuracies can arise, consists of the measurement of the defect vector. The subsequent steps of calculating the true position of the defect can be carried out entirely by computer using accurate and well-understood formulas. If these algorithms are correctly implemented they should not introduce any errors apart from minor inaccuracies due to rounding off of numbers.

Measurement of the defect vector requires the observer to estimate the distance to, and bearing of, the defect. At the same time the observer's position is recording using a GPS receiver. All three of these steps are subject to inaccuracy. Of the three, estimating distances is the most difficult at relies entirely on the ability of the observer to do so accurately. While this ability can be improved through training and experience, some observers will always be better at judging distances than others. However, given the typical distances and defect sizes that will be encountered in a mine, the error introduced by estimating distances is not considered to be a serious hindrance. For example, for a haul road that is 40 metres wide, an observer would be able to get to within 40 metres of a defect even if the defect were on the far side of the road. If the observer's estimate of the distance to the defect was out by $10 \%$, i.e. 4 metres, this would still be smaller than the positional error of typical modern hand-held GPS receivers, which have accuracies of approximately 10 metres. Furthermore, it is also smaller than the areal extent of most defects. Distance errors can be eliminated by using laser range finders but this defeats the main reason for developing this low cost and easily implementable defect measuring system.

Determining the bearing to the defect is also subject to error, although, as with the estimation of distance, this should improve with experience. The measurement of defect bearings can also made easier by using a hand-held compass. The observer would need to align the compass dial so that the North reading $\left(0^{\circ}\right)$ points to the left and the South reading $\left(180^{\circ}\right)$ to the right. The defect bearing is then read off from the compass dial. Note that for the purposes of measuring the defect bearing, the direction in which the compass needle points is irrelevant as it is the defect bearing relative to the road that is of importance, not the bearing relative to Magnetic North.

The accuracy of the third step in defining the defect vector, namely recording the defect vector origin using a GPS receiver, can be improved by using methods such as 
differential correction. Once again, this would lead to increased costs.

Although the three components of the defect vector are all subject to measurement error, these are generally smaller than the sizes of the defects being measured. What is required from the methodology outlined above is that maintenance teams be able to locate defects using the defect position calculated using the defect vector method. Given the areal extent of typical haul road defects, guiding maintenance crews to within ten metres of the defect would allow them to see the defect anyway.

\section{REFERENCES}

[1] L. P. Sicking, "Rangefinder comparison", http://www.fs.fed.us/ eng/pubs/html/98241307/98241307.html

\section{AUTHORS}

K. J. Duffy is the Director of the Centre for Systems Research (CSR) at the Durban University of Technology in Durban, South Africa (e-mail: kevind@ dut.ac.za). He has a Ph.D. from the University of Virginia, USA.

M. Rawlins is CEO of Energy Combustion Services (ECS) in Durban (e-mail: mark.rawlins@enerserv.co.za). He has a Doctor of Technology degree from the Durban University of Technology.

F. Sokolic is a consultant with GI Solutions in Durban and works with both ECS and the CSR (e-mail: sokolic@worldonline.co.za). He has an M.Sc. from the University of KwaZulu-Natal in Durban.

This work was supported in part by the South African National Research Foundation's Technology and Human Resources for Industry Programme.

Submitted, September, 28, 2009. Published as resubmitted by the authors on December, 02, 2009. 